\title{
Effect of Introducing the Mucosal Atomization Device for Fentanyl Use in Out-of-Hospital Pediatric Trauma Patients
}

\author{
Daniel P. O'Donnell, MD; Luke C. Schafer, MD; Andrew C. Stevens, MD; \\ Elizabeth Weinstein, MD; Charles M. Miramonti, MD; Mary Ann Kozak, DrPH
}

Department of Emergency Medicine, Indiana University School of Medicine, Indianapolis, Indiana USA

\section{Correspondence:}

Daniel P. O’Donnell, MD

Department of Emergency Medicine

Indiana University School of Medicine

3930 Georgetown Rd.

Indianapolis, Indiana 46254 USA

E-mail: dapodonn@iupui.edu

Conflicts of interest: The authors have no relevant financial information or potential conflicts of interest to disclose.

Keywords: device; Emergency Medical Services; fentanyl; intranasal; pain assessment; pediatric; trauma

\section{Abbreviations:}

EMS: Emergency Medical Service

MAD: mucosal atomization device

Received: October 5, 2012

Revised: February 1, 2013

Accepted: February 13, 2013

Online publication: May 24, 2013

doi:10.1017/S1049023X13003579

\begin{abstract}
Background: Pain associated with pediatric trauma is often under-assessed and undertreated in the out-of-hospital setting. Administering an opioid such as fentanyl via the intranasal route is a safe and efficacious alternative to traditional routes of analgesic delivery and could potentially improve pain management in pediatric trauma patients. Objective: The study sought to examine the effect of introducing the mucosal atomization device (MAD) on analgesia administration as an alternative to intravenous fentanyl delivery in pediatric trauma patients. The hypothesis for the study is that the introduction of the MAD would increase the administration of fentanyl in pediatric trauma patients. Methods: The research utilized a 2-group design (pre-MAD and post-MAD) to study 946 pediatric trauma patients (age <16) transported by a large, urban EMS agency to one of eight hospitals in Marion County, which is located in Indianapolis Indiana. Two emergency medicine physicians independently determined whether the patient met criteria for pain medication receipt and a third reviewer resolved any disagreements. A comparison of the rates of fentanyl administration in both groups was then conducted. Results: There was no statistically significant difference in the rate of fentanyl administration between the pre-MAD (30.4\%) and post-MAD groups $(37.8 \%)(P=.238)$. A subgroup analysis showed that age and mechanism of injury were stronger predictors of fentanyl administration.

Conclusion: Contrary to the hypothesis, the addition of the MAD device did not increase fentanyl administration rates in pediatric trauma patients. Future research is needed to address the barriers to analgesia administration in pediatric trauma patients.
\end{abstract}

O’Donnell DP, Schafer LC, Stevens AC, Weinstein E, Miramonti CM, Kozak MA. Effect of introducing the mucosal atomization device for fentanyl use in out-of-hospital pediatric trauma patients. Prehosp Disaster Med. 2013;28(5):520-522.

\section{Introduction}

Health care providers often do a poor job assessing and treating pain in pediatric patients. ${ }^{1-4}$ This oligoanalgesia is recognized by the American Academy of Pediatrics which recommends using all available methods of pain management to eliminate barriers to analgesic administration in children. ${ }^{5}$ In the out-of-hospital setting, perceived patient discomfort and the difficulty associated with intravenous access are thought to be large barriers to appropriate pain management in pediatric patients. The delivery of intranasal analgesia may be a novel solution to this problem. ${ }^{6}$ Intranasal fentanyl is both safe and efficacious in the management of acute painful conditions associated with pediatric trauma. $^{7}$ The study investigated whether the introduction of the mucosal atomization device as an additional method for fentanyl delivery (MAD, Model 300, Wolf Tory Medical, Salt Lake City, Utah, USA) would increase fentanyl administration rates in pediatric trauma patients.

Methods

Study Design

The research used a 2 -group, retrospective study of pediatric trauma patients (age $<16)$ transported to one of eight hospitals by an urban, hospital-based ambulance service during 
a 13-month period. Study Group 1 included all pediatric trauma patients transported during the 6-month period before introducing the MAD (September 1, 2007 through February 28, 2008). During this period, the only delivery method for fentanyl was intravenous. In March 2008, a training session was conducted to educate paramedics about the new device in conjunction with a revised county EMS protocol. Study Group 2 included all pediatric trauma patients transported during the succeeding 6-month period, April 1, 2008 through September 30, 2008. During the second period, the paramedic could administer fentanyl intravenously or via the MAD device.

\section{Study Setting and Populations}

The setting was an urban, hospital-based ambulance service with an annual call volume of over 80,000 providing the majority of out-of-hospital care for the city of Indianapolis, located in Marion County, Indiana. The study populations included two groups of pediatric trauma patients identified as candidates for pain medication using the county EMS pain management protocol. Prior to introduction of the MAD device, all providers were mandated to undergo a 3-hour classroom session on the revised EMS protocols. One component of this session included an in-service training on the use of the MAD device as an alternative for fentanyl administration. The training focused on the motor skills necessary for proper use of the MAD while giving some background on the efficacy of intranasal fentanyl administration. Providers were not encouraged to favor one route over the other. Once this training was completed, all providers were permitted to use the MAD device as a method for fentanyl delivery. The route of delivery as well as the decision to administer was made by the treating paramedic. In accordance with the protocol, fentanyl was indicated when a patient was describing moderate to severe pain which was defined as a pain rating greater than 3 on a 1 to 10 scale or a score greater than 4 on the Wong-Baker FACES pain rating scale. In Group 1, fentanyl was administered intravenously, the standard of care, and in Group 2, fentanyl could be administered intravenously or via the MAD. Inclusion criteria were all pediatric trauma patients older than 15 years of age. Patients were excluded if they had a verbal pain score of less than 3 out of 10, Wong-Baker score less than 4, or Glasgow Coma Scale (GCS) of less than 14. The Indiana University School of Medicine Institutional Review Board (IRB) categorized the study as exempt from IRB review.

\section{Measurements}

Two emergency physicians individually reviewed each chart to determine appropriateness for fentanyl administration using the Marion County EMS pain management protocol. Since a pain scale was not a required entry for pediatric patients, charts were reviewed for documented evidence that the patient was experiencing pain; for example, the patient was described to be in moderate to severe discomfort, inconsolable, or had an obvious deformity. Conversely, if there was evidence the patient was wellappearing and in no acute distress, the reviewer determined that fentanyl was not indicated. A third, independent, physician reviewer resolved any discrepancies between the initial reviewers. A Kappa ${ }^{8}$ score was computed to reflect agreement between the initial reviewers. A research assistant recorded vital signs, mechanism of injury, and method and rate of fentanyl administration from the out-of-hospital record. Trauma was categorized as an ICD-9 code between 900 and 959.9, and the mechanism of injury was indicated using a short description of how the child was injured. The research assistant then organized mechanism of injury into seven categories.

\section{Data Analysis}

Two-sample $t$ tests and chi-square tests were used to identify differences in demographic and clinical characteristics by time period and fentanyl administration. SAS Version 9.3 (SAS Institute, Cary, North Carolina, USA) was used for all analyses.

\section{Results}

A total of 946 patient-care records were reviewed-518 in the pre-MAD group and 428 in the post-MAD group. In the preMAD group, of the 518 charts reviewed, 135 patients $(26.1 \%)$ met the criteria for pain medication administration; of these, $41(30.4 \%)$ received fentanyl. In the post-MAD group, of the 428 charts reviewed, 98 patients $(22.9 \%)$ met the criteria, and of these, $36(36.7 \%)$ received fentanyl. Only 13 (36.1\%) patients of those receiving fentanyl were treated with the MAD. Using the Kappa score, reviewer agreement for both the pre and post MAD groups was $86.7 \%$ (95\% CI, 0.811-0.924).

The mean age, in years, of both the pre-MAD group and the post-MAD group was identical (9.9, SD 4.5). Both groups were predominantly male. There was no difference in the mean Glasgow Coma Scale or mean initial pulse. The difference between the mean initial respiratory rate was significant: 22.3 (SD 6.1) in the pre-MAD group and 20.6 (SD 5.3) in the postMAD group, $P<.05$.

Males tended to receive fentanyl more than females in both the pre- and post-MAD groups (37.9\% vs $26.9 \%)$. However, this trend was not statistically significant. Furthermore, there was a trend toward older children receiving fentanyl when compared with younger children. Just over $23 \%$ of those receiving fentanyl were $0-5$ years of age; $27.6 \%$ were $6-10$ years of age; and $39.8 \%$ were 11-15 years of age. Again, while there was a trend, it did not reach statistical significance. Mechanism of injury, however, was a statistically significant predictor of fentanyl administration. The percentage of children receiving fentanyl by mechanism of injury was assault, $16.7 \%$; burn, $69.2 \%$; cut, $20.0 \%$; fall, $40.9 \%$; motor vehicle crash, $13.0 \%$; struck by or against, $33.3 \%$; and other, $33.6 \% ; P<.001$. Injuries in the "other" category included animal bite, bicycle, pedestrian, penetrating, or sports injury.

The logistic regression analysis of the factors influencing fentanyl use is presented in Table 1. As age increased, the likelihood of receiving fentanyl increased. Similar results were found for initial pulse of the patient. Burn patients were associated with an increase in fentanyl use in the post-MAD period (adjusted odds $=6.34 ; 95 \% \mathrm{CI}, 1.14-35.41$ ) while motor vehicle crash patients were associated with a decrease in fentanyl use in the post-MAD period (odds $=0.29 ; 95 \% \mathrm{CI}, 0.09-0.89$ ).

\section{Discussion}

This study indicates there was no significant difference in fentanyl administration among the pre- and post-MAD groups. These results were in contrast to the study hypothesis that this alternative delivery method would improve the rate of fentanyl administration. It is unclear why paramedics did not use the MAD as expected given that intravenous access has been perceived as a barrier to fentanyl administration and administering fentanyl via the intranasal route is comparatively easy and painless. 


\begin{tabular}{|c|c|c|}
\hline Factor & Odds Ratio & $95 \% \mathrm{Cl}$ \\
\hline Post-MAD & 1.75 & $0.86-3.66$ \\
\hline Male vs female patient & 1.15 & $0.60-2.22$ \\
\hline \multicolumn{3}{|l|}{ Age, y } \\
\hline $0-5$ vs $11-15$ & 0.10 & $0.03-0.36$ \\
\hline $6-10$ vs $11-15$ & 0.39 & $0.18-0.86$ \\
\hline Initial RR & 0.99 & $0.92-1.07$ \\
\hline Initial GCS & 1.34 & $0.58-3.10$ \\
\hline Initial pulse & 1.02 & $1.01-1.04$ \\
\hline \multicolumn{3}{|l|}{ Mechanism of injury } \\
\hline Assault vs other & 0.19 & $0.03-1.13$ \\
\hline Blunt vs other & 1.06 & $0.28-4.50$ \\
\hline Burn vs other & 6.34 & $1.14-35.41$ \\
\hline Cut vs other & 0.36 & $0.06-2.28$ \\
\hline Fall vs other & 1.70 & $0.71-4.08$ \\
\hline MVC vs other & 0.29 & $0.09-0.89$ \\
\hline
\end{tabular}

Table 1. Logistic Regression of Factors Influencing Fentanyl Use in Pediatric Patients

Abbreviations: RR, Respiratory Rate, GCS, Glasgow Coma Scale; $\mathrm{MAD}$, mucosal atomization device; MVC, motor vehicle crash

Logistic regression results from the current study confirm that fentanyl use in the post-MAD period was associated with older children, specifically those between the ages of 11 and 15 years. In fact, as age decreased, the likelihood of receiving fentanyl in the post-MAD period decreased such that children 0-5 years of age were associated with less fentanyl than the 6-10 age group, even though the hypothesis was that the MAD would increase fentanyl use in the younger population. These results are similar to a study by Swor $^{2}$ concluding that younger children with painful conditions such as an extremity fracture receive analgesia less frequently when compared with older children with similar injuries. Also similar to Swor, ${ }^{2}$ fentanyl use in the post-MAD

\section{References}

1. American Academy of Pediatrics. Committee on Psychosocial Aspects of Child and Family Health; Task Force on Pain in Infants, Children, and Adolescents. The assessment and management of acute pain in infants, children, and adolescents. Pediatrics. 2001;108(3):793-797.

2. Swor R, McEachin CM, Seguin D, Grall KH. Prehospital pain management in children suffering traumatic injury. Prehosp Emerg Care. 2005;9(1):40-43.

3. Alexander J, Manno M. Underuse of analgesia in very young pediatric patients with isolated painful injuries. Ann Emerg Med. 2003;41(5):617-622.

4. Garrison HG, Maio RF, Spaite D, et al. Emergency Medical Services Outcomes Project (EMSOP) IV: pain measurement in out-of-hospital outcomes research. Ann Emerg Med. 2002;40(2):172-179.

5. Zempsky WT, Cravero JP. American Academy of Pediatrics Committee on Pediatric Emergency Medicine and Section on Anesthesiology and Pain Medicine. Relief of pain and anxiety in pediatric patients in emergency medical systems. Pediatrics. 2004;114(5):1348-1356. period was associated with burns and not associated with motor vehicle crashes.

Zempsky ${ }^{5}$ and colleagues hypothesize that it is possible that paramedics do not assess or manage pain in pediatric trauma patients due to a lack of adequate assessment tools. In a survey by Hennes ${ }^{9}$ of paramedics in a large urban ambulance service, only $6 \%$ and $31 \%$ stated they had used a verbal pain scale in children and adolescents, respectively, in the previous month. Further, a study by Izsak $^{10}$ of nearly 700 pediatric trauma patients transported by a county ambulance service identified only one pediatric trauma chart documenting that a validated pain assessment tool was used. This study had similar findings. Many of the charts reviewed had no documented pain score. These studies suggest that a major limitation to pain management is proper assessment rather than difficult intravenous delivery. However, it is also possible that paramedics did not easily embrace this new alternative delivery method due to the fact that intranasal drug delivery had never been done prior to this protocol change. This change could be due to the difficulty of implementing a new method when paramedics are entrenched in the traditional method of medication administration.

\section{Limitations}

There are a few limitations for this study. First, only one ambulance service was studied, thus limiting the study's external validity. Second, this study was conducted over two successive 6-month periods and does not reflect the seasonal nature of trauma. To obtain a complete picture of the rate of injury in pediatric trauma patients, 12 months of data should have been collected for both the pre- and post-MAD groups. However, this study was a pilot study on the use of fentanyl by out-of-hospital care providers. Third, the paramedics had a limited opportunity to use the device based on the small number of pediatric trauma patients in both groups. Therefore, use of the MAD reflects only a portion of the paramedics' experience with the MAD.

\section{Conclusion}

The results of this study indicate that fentanyl use is not statistically different between the pre- and post-MAD groups. Given that fentanyl administration in this population of pediatric trauma patients was limited to $34.8 \%$ of total patients transported, additional research is needed to identify methods that will improve the assessment and treatment of pain in out-of-hospital pediatric trauma patients.

6. Borland ML, Jacobs I, King B, O’Brien D. A randomized controlled trial comparing intranasal fentanyl to intravenous morphine for managing acute pain in children in the emergency department. Ann Emerg Med. 2007;49(3):335-340.

7. Borland ML, Jacobs I, Geelhoed G. Intranasal fentanyl reduces acute pain in children in the emergency department: a safety and efficacy study. Emerg Med (Fremantle). 2002;14(3):275-280.

8. Fleiss JL. Statistical Methods for Rates and Proportions. New York: John Wiley \& Sons; 1981.

9. Hennes H, Kim MK, Pirrallo RG. Prehospital pain management: a comparison of providers' perceptions and practices. Prehosp Emerg Care. 2005;9(1):32-39.

10. Izsak E, Moore JL, Stringfellow K, Oswanski MF, Lindstrom DA, Stombaugh HA. Prehospital pain assessment in pediatric trauma. Prehosp Emerg Care. 2008;12(2): 182-186. 